\title{
Prospective open-label evaluation of long-term low-dose doxycycline for difficult-to-treat chronic rhinosinusitis with nasal polyps*
}

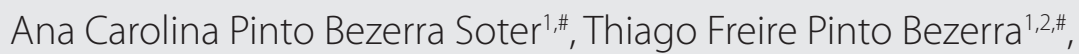 \\ Rogério Pezato 1,2, Tatiana Regina Teles Abdo', Renata Mendonça Pilan', \\ Fabio de Rezende Pinna', Philippe Gevaert ${ }^{3}$, Thibaut van Zele³, \\ Claus Bachert ${ }^{3}$, Richard Louis Voegels ${ }^{1}$ \\ 'Department of Otolaryngology and Ophthalmology, University of Sao Paulo School of Medicine, São Paulo, Brazil \\ 2 Departament of Surgery, Federal University of Pernambuco, Hospital das Clínicas (UFPE), Cidade Universitária, Recife, Brazil \\ ${ }^{3}$ Upper Airway Research Laboratory (URL), Department of Otorhinolaryngology, Ghent University Hospital, Ghent University, \\ Ghent, Belgium
}

Rhinology 55: 175-180, 2017

https://doi.org/10.4193/Rhino15.291

*Received for publication:

October 21, 2015

Accepted: March 11, 2017

\# these authors contributed equally to this work

\begin{abstract}
Background: This study aimed to assess clinical outcomes of long-term low-dose oral doxycycline therapy in difficult-to-treat chronic rhinosinusitis with polyps (CRSwNP).
\end{abstract}

Methods: This was a prospective, open-label study of 60 patients with difficult-to-treat CRSwNP who had undergone endoscopic sinus surgery. Patients were divided into two groups: 28 received nasal steroids, saline irrigation, and doxycycline (200 mg on the first day, followed by $100 \mathrm{mg}$ once daily) for 12 weeks, while 30 received only nasal steroids and saline irrigation. The main outcome measure was an adequate effect size of doxycycline treatment on clinically meaningful significant improvement of SNOT-20. Other outcome measures were the SNOT-20, NOSE, and Lund-Kennedy scores. The following parameters were also analyzed: asthma, rhinitis, non-steroidal-exacerbated respiratory disease (NERD), and baseline serum $\lg G, \lg A$, $\lg E$, $\lg M$, ANCA, and eosinophil count.

Results: There was an adequate effect size of doxycycline treatment on clinically meaningful significant improvement of SNOT-20. Patients who received doxycycline also had significantly better outcomes regarding SNOT-20, NOSE, and Lund-Kennedy scores. There was a negative association among a clinically significant improvement of SNOT-20 and presence of asthma, NERD, and elevated serum lgE levels before treatment.

Conclusion: These findings suggest that doxycycline may have a beneficial role for CRSWNP patients, especially for patients without asthma, NERD or high levels of serum IgE before treatment.

Key words: nasal polyps, sinusitis, doxycycline, quality of life, antibiotic

\section{Introduction}

Chronic rhinosinusitis (CRS) can be described as a dysfunctional host-environment interaction at the site of interface, which occurs in the nose and paranasal sinuses. The overall prevalence of CRS is estimated at 14\% in the United States, $10.9 \%$ in Europe, and $5.5 \%$ in the city of São Paulo, Brazil ${ }^{(1-4)}$. Chronic rhinosinusitis with nasal polyps (CRSwNP) is a particular subset of CRS characterized by a mucosal inflammation that leads to mucosal thickening and polyp formation, and can be especially difficult to treat. Patients with CRSwNP report a great negative impact on quality of life (QoL) ${ }^{(5)}$. Patients who do not achieve acceptable control of CRS despite adequate surgery, intranasal corticosteroid treatment, and up to two short courses of antibiotics or systemic corticosteroids in the last year are considered to have 
difficult-to-treat rhinosinusitis ${ }^{(1)}$.

The pathophysiology of CRSwNP remains largely unknown ${ }^{(1,6,7)}$. In terms of inflammatory response, the majority of Caucasian patients with CRSwNP present a T-helper cell type 2 (Th2) cytokine profile, eosinophil-mediated inflammation, decreased concentrations of transforming growth factor-beta (TGF- $\beta$ ), and elevated levels of matrix metalloproteinase-9 (MMP-9) (8-11). Another factor consistently related with a subgroup of CRSwNP patients is the interaction of Staphylococcus aureus with Thelper lymphocytes, which independently modulates the adaptive immune response through biofilm formation or superantigen production ${ }^{(12-15)}$. Moreover, a higher risk of clinical and microbiological late relapse is present when Staphylococci coexist as biofilms on the sinus mucosa and as intracellular bacteria ${ }^{(15)}$. Biofilm presence per se is not associated with poorer prognosis, and biofilms have been found both in CRSwNP patients and over the normal ciliated sinus mucosa of controls ${ }^{(16,17)}$. Conversely, presence of Staphylococcus aureus in the biofilm is associated with poorer prognosis as well as with the worst clinical profile of CRS, a finding attributable to modulation of inflammatory activity by staphylococcal superantigens ${ }^{(18)}$.

Doxycycline is an orally available, broad-spectrum bacteriostatic antibiotic of the tetracycline class, which also has anti-inflammatory action ${ }^{(19-21)}$. MMP-9 levels in nasal secretions are significantly reduced after doxycycline administration, a fact that could alter the remodeling process and, consequently, may act on the mechanical dysfunction found in nasal polyposis ${ }^{(22)}$. The ability of doxycycline to reduce eosinophil cationic protein (ECP) reflects downregulation of eosinophil degranulation, whereas the reduction of myeloperoxidase levels after doxycycline administration reflects reduced neutrophilic activity ${ }^{(20)}$. This drug could act at some of the above-listed targets. Furthermore, doxycycline has antibiotic activity against Staphylococcus aureus within polymorphonuclear neutrophils ${ }^{(21)}$. This fact probably will help to reduce superantigen production. van Zele et al. showed that administration of doxycycline for 20 days significantly reduced polyp size in a similar effect to that observed after 4 weeks of therapy with mometasone furoate ${ }^{(20)}$. It also significantly reduced postnasal drip, and longer-term treatment or higher doses would be expected to improve symptoms and outcomes. In the current study, we tested the hypothesis that long-term low-dose oral doxycycline therapy could improve clinical control of difficult-to-treat CRSwNP.

\section{Materials and methods}

This was a single center, non-stratified (adults older than 18 years), with balanced randomization [1:1], open label, trial, nonplacebo-controlled study conducted between 2013 and 2014 at an university-based tertiary care center (University of São Paulo School of Medicine, São Paulo, Brazil). It was partially funded by FORL (Fundação Otorrinolaringologia da Universidade de São
Paulo). There is not any changes to methods after trial commencement. Block-randomization was performed and the first 30 patients were randomly computer assigned to Doxycycline Group.

The local ethics committee approved the study and provided written informed consent for participation. This study was registered at National Registry (07120413.3.0000.0068)

The inclusion criteria were a diagnosis of CRSwNP according to the EPOS definition with bilateral endoscopic evidence of nasal polyps ${ }^{(1)}$ and persistent symptoms of rhinosinusitis despite appropriate treatment (recommended medication and surgery) at least 12 months after surgery. Functional endoscopic sinus surgery was extended into all paranasal sinuses presumed to be diseased according to nasal endoscopy or CT scan findings. Radical procedures, such as nasalization, were not performed in any patient. The medication prescribed to all patients during the year after surgery was isotonic saline irrigation $(20 \mathrm{cc} /$ nostril b.i.d) .and topical nasal steroid sprays (fluticasone propionate spray, $100 \mathrm{mcg} /$ nostril b.i.d., starting at least 3 weeks after surgery). A 3-month washout period without the use of any medication other than those described above was established. Systemic corticosteroids or local or systemic antibiotic therapy were not allowed during the study. Diagnosis of allergy was based on ARIA guidelines and skin prick testing. Diagnosis of asthma was based on the American Thoracic Society classification, and diagnosis of non-steroidal-exacerbated respiratory disease (NERD) was based on clinical history1 (Figure 1).

The exclusion criteria were: secondary causes of CRS, sinonasal malignancy, radiation therapy to the head and neck, craniofacial syndromes, anterior nasal trauma or fracture, adenoid hypertrophy, sarcoidosis, granulomatosis of the nasal cavity, uncontrolled asthma, pregnancy, and immunodeficiency.

\section{Study protocol}

Sixty eligible patients were randomly assigned to one of two groups by order of inclusion. During the study, all patients received nasal isotonic saline irrigation and topical nasal steroids (fluticasone propionate spray $100 \mathrm{mcg} /$ nostril b.i.d.). Half of the patients were randomized to also receive doxycycline (200 mg on the first day, followed by $100 \mathrm{mg}$ once daily) for 12 weeks after a 3-month washout period. A follow-up visit was scheduled for 12 weeks after the start of treatment.

Baseline assessments included full medical history, QoL assessment with SNOT-20 ${ }^{(23-25)}$ and NOSE ${ }^{(26,27)}$, and ENT examination. Patients also were evaluated for concomitant diagnoses of asthma, rhinitis, and NERD. All patients underwent nasal endoscopic examination (graded according to Lund-Kennedy score ${ }^{(28)}, \mathrm{CT}$ scan (graded according to Lund-Mackay score ${ }^{(28)}$, and peripheral blood testing for $\lg G, \lg A$, $\lg E$, IgM, ANCA, and eosinophil count. 


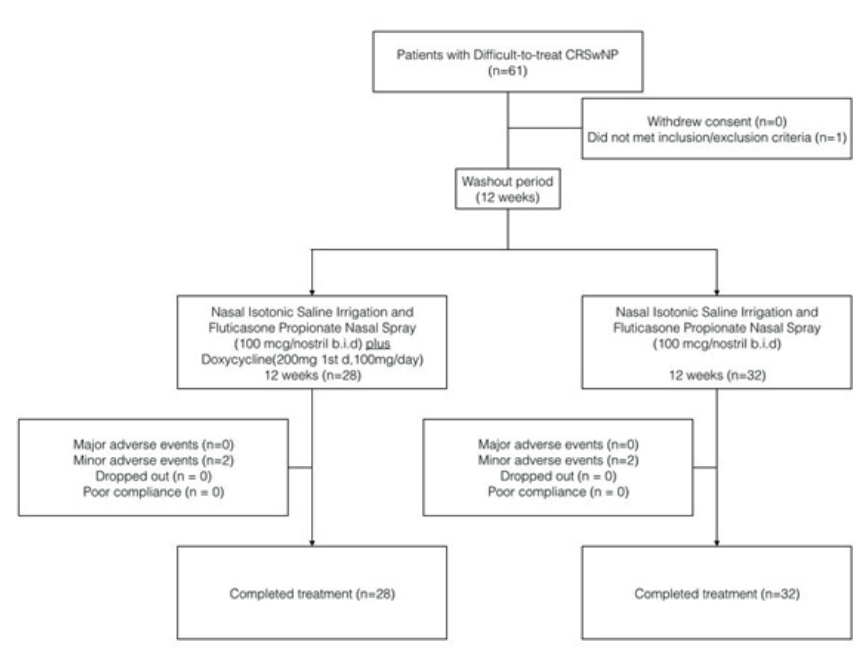

Figure 1. Participant flow diagram.

\section{Outcome measures}

The key outcome measure was a clinically meaningful improvement in disease-specific QoL as evaluated by SNOT-20. This improvement was defined as a $>0.8$ reduction in SNOT-20 total score, as established in the literature ${ }^{(25)}$. The secondary outcome measures were not blinded endoscopic scores (Lund-Kennedy), quality-of life disease-specific questionnaires for rhinosinusitis (SNOT-20) and Nasal Obstruction (NOSE). Sides effects were previously read on informed consent and promptly asked every office visit. A 24/7 Telephone Number and Address was provided

\section{Statistical analysis}

A power analysis predicted that sample size of 22 in each group would be required to achieve $80 \%$ power at the $5 \%$ level of significance. A minimal sample size of 30 patients per group was necessary, given an anticipated dropout rate of $25 \%$.

The Kolmogorov-Smirnov test demonstrated nonparametric distribution of the data, which were thus expressed as medians and interquartile ranges. The Mann-Whitney $\mathrm{U}$ test was used to compare values between groups. Dichotomous data were analyzed by the chi-square or Fisher's exact test.

We evaluated the effect size of Doxycycline treatment on Clinically significant improvement of SNOT-20 (reduction higher than 0.8 ) by classic and intention-to-treat odds ratio. The intention-totreat analysis of odds ratio included patients whose refused to participate as a negative outcome. Statistical analysis of efficacy was based on all subjects included in the study.

All statistical analyses were performed in the SPSS 17.0 software environment (SPSS, Inc., Chicago, IL, USA). For all statistical tests,

Table 2. Mental well-being variables by CRS group.

Doxycycline + nasal steroids Median (IQR)

Nasal steroids alone Median (IQR)

\begin{tabular}{|lccc}
\hline Gender (M:F) & $13: 15(0.87)$ & $14: 16(1)$ & 0.99 \\
\hline Age, years & $47.50(16)$ & $47.50(14)$ & 0.73 \\
\hline Rhinitis & $28.6 \%(8 / 28)$ & $40.0 \%(12 / 30)$ & 0.36 \\
\hline Asthma & $57.1 \%(16 / 28)$ & $50.0 \%(18 / 30)$ & 0.82 \\
\hline NERD & $39.3 \%(11 / 28)$ & $26,7 \%(8 / 30)$ & 0.31 \\
\hline SNOT-20 (pre-treatment) & $1.77(1.35)$ & $2.05(1.46)$ & 0.36 \\
\hline NOSE (pre-treatment) & $50.00(68)$ & $52.50(70)$ & 0.18 \\
\hline Lund-Kennedy (pre-treatment) & $10(3)$ & $11(4)$ & 0.21 \\
\hline Lund-Mackay (pre-treatment) & $18(6)$ & $17(8)$ & 0.36 \\
\hline Eosinophils (absolute count) & $300(275)$ & $400(500)$ & 0.26 \\
\hline Eosinophils (\%) & $4.9(3.7)$ & $5.1(6.5)$ & 0.56 \\
\hline IgA & $278(128)$ & $289(104)$ & 0.98 \\
\hline Normal IgE & $53.6 \%(15 / 28)$ & $46,7 \%(14 / 30)$ & 0.60 \\
\hline IgE & $141(245)$ & $218(401)$ & 0.53 \\
\hline IgG & $1162.50(452)$ & $1202.00(425)$ & $141(103)$ \\
\hline IgM & $117(75)$ & 0.99
\end{tabular}

NERD, aspirin-exacerbated respiratory disease; Lund-Kennedy score for nasal endoscopic evaluation; Lund-Mackay score for CT scan evaluation; IQR, interquartile range; NOSE, Nasal Obstruction Symptom Evaluation; SNOT-20 - Sino-Nasal Outcome Test-20. 
Table 2. Clinical outcomes stratified by group allocation.

\begin{tabular}{|lccc|}
\hline & $\begin{array}{c}\text { Doxycycline }+ \\
\text { nasal steroids } \\
\text { Median (IQR) }\end{array}$ & $\begin{array}{c}\text { Nasal steroids } \\
\text { alone Median } \\
\text { (IQR) }\end{array}$ & p \\
\hline $\begin{array}{l}\text { SNOT-20 outcome } \\
\begin{array}{l}\text { Clinically signifi- } \\
\text { cant improvement } \\
\text { of SNOT-20 }\end{array}\end{array}$ & $-0.85(0.84)$ & $-0.45(0.33)$ & $0.002^{*}$ \\
$\begin{array}{l}\text { NOSE outcome } \\
\begin{array}{l}\text { Lund-Kennedy } \\
\text { outcome }\end{array}\end{array}$ & $-15.0(37.5)$ & $-7.5(16.25)$ & $0.043^{*}$ \\
\hline
\end{tabular}

Lund-Kennedy score for nasal endoscopic evaluation; Lund-Mackay score for CT scan evaluation; IQR, interquartile range; NOSE, Nasal Obstruction Symptom Evaluation; SNOT-20 - Sino-Nasal Outcome Test20.

$p=0.05$ was considered statistically significant.

\section{Results}

A total of 60 patients were evaluated: 28 patients received the study intervention (nasal steroids plus doxycycline for 12 weeks), while 30 patients received only nasal steroids (Table 1). Two patients allocated to doxycycline group refused to participate and were analyzed in the nasal steroids group. Baseline (pre-treatment) median SNOT-20 scores did not differ significantly between the intervention and comparator groups (1.77 [1.35] vs. 2.05 [1.46], respectively, $p=0.47$ ), (Figure 2). The two groups were also homogeneous for other parameters of interest at baseline (Table 1).

None of the patients in either group withdrew from the study, and no patient experienced major adverse events. No patient had to drop out due to acute exacerbation of CRS or asthma. Only four patients experienced minor adverse events (two in each group, all during the first days of treatment), but none had to discontinue therapy. These four patients presented watery nasal discharge and nasal itching, controlled with 1 day of antihistamine, and one in the doxycycline group experienced an acute exacerbation of asthma, controlled with a brief ( 5 days) course of beta2 agonist therapy.

\section{Clinical efficacy}

After 12 weeks, there was a statistically significant association between meaningful improvement in SNOT-20 score and doxycycline treatment $(50.0 \%$ [14/28] vs $10.0 \%$ (3/30), $p=0.01$; $\mathrm{OR}=7.87 \mathrm{IC} 95 \%: 1.96-31.67, \mathrm{p}=0.003$. The patients who received doxycycline also showed statistically significant responses in SNOT-20 ( $p=0.002)$, NOSE ( $p=0.046)$, and Lund-Kennedy scores $(p=0.004)$ (Table 2 ).

Patients who exhibited clinically significant improvement in

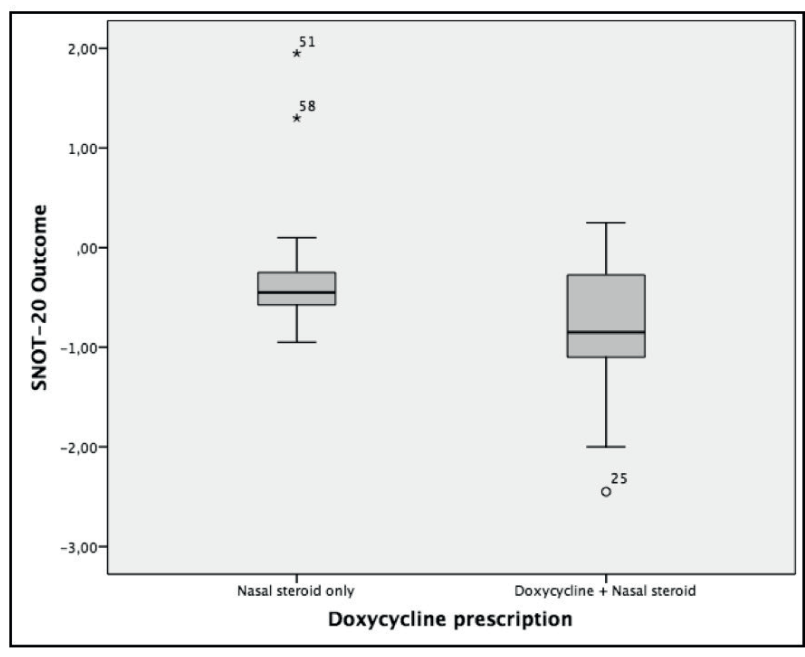

Figure 2. Comparison of SNOT-20 scores between the intervention (doxycycline + nasal steroid) and comparator (nasal steroid alone) groups.

SNOT-20 also presented better outcomes on total SNOT-20 and NOSE scores ( $p=0.001)$, as well as near-significant improvement in Lund-Kennedy scores $(p=0.07)$. Asthma $(p=0.006)$, NERD $(p=0.018)$, and elevated serum $\lg E(p<0.035)$ at baseline were associated with worse prognosis after treatment, shown by a non-clinically significant improvement of SNOT-20 in these subgroups. Patients who did not achieve clinically significant improvement with the study intervention had significantly worse endoscopic scores at baseline $(p=0.016)$ (Table 3 ).

\section{Discussion}

The findings of this report suggest that doxycycline is a good option to improve QoL in a portion of patients with difficult-totreat CRSwNP without response to any classic treatment modalities. Doxycycline treatment improved disease-specific QoL outcomes related to sinonasal symptoms $(p<0.05)$ and nasal obstruction $(p<0.04)$. Moreover, we found that $50.0 \%$ of patients (14 of 28) experienced clinically meaningful improvement in SNOT-20 scores $(p<0.05)$ after doxycycline treatment. The effect of doxycycline on improvement of SNOT-20 score in CRSwNP was particularly evident in patients with normal serum IgE levels $(p<0.01)$ and normal eosinophil counts $(p<0.05)$ before treatment. These findings are corroborated by the lower prevalence of asthma $(p<0.01)$ and NERD $(p<0.01)$ in the subset of patients who showed clinically significant improvement after treatment. We found also higher pre-treatment lgG levels in patients who improved $(p<0.05)$. We did not analyze any inflammatory markers in nasal secretions, but van Zele et al. previously revealed that doxycycline reduced MMP-9, myeloperoxidase, and ECP levels ${ }^{(20)}$. Although van Zele et al. did not find any relationship of $\lg$ E level or eosinophil count with clinical outcome after 20 days of doxycycline therapy, long-term clarithromycin treatment was associated with better results in CRS patients with normal 
Table 3. Patient characteristics stratified by presence or absence of clinically significant improvement in SNOT-20 score.

\begin{tabular}{|c|c|c|c|}
\hline & $\begin{array}{l}\text { Clinically significant improvement of } \\
\text { SNOT-20 (+) }\end{array}$ & $\begin{array}{l}\text { Clinically significant improvement of } \\
\text { SNOT-20 (-) }\end{array}$ & $\mathrm{p}$ \\
\hline Gender (M:F) & $5: 9(0.56)$ & $8: 6(1.33)$ & 450 \\
\hline Age & $43.00(21)$ & $48.00(14)$ & 0.173 \\
\hline SNOT-20 (pre-treatment) & $2.28(1.38)$ & $1.55(1.11)$ & 0.094 \\
\hline NOSE (pre-treatment) & $65.00(46)$ & $37.50(58)$ & 0.210 \\
\hline Lund-Kennedy (pre-treatment) & $8.50(4)$ & $10.50(3)$ & $0.016^{*}$ \\
\hline Lund-Mackay (pre-treatment) & $16.00(7)$ & $18.50(5)$ & 0.137 \\
\hline SNOT-20 (outcome) & $-0.96(0.46)$ & $-0.28(0.64)$ & $0.001^{*}$ \\
\hline NOSE (outcome) & $-30.00(26.5)$ & $0.00(26.5)$ & $0.001^{*}$ \\
\hline Lund-Kennedy (outcome) & $-4.00(3.25)$ & $-3.00(1.50)$ & 0.070 \\
\hline Rhinitis & $28,6 \%(4 / 14)$ & $28,6 \%(4 / 14)$ & 1.00 \\
\hline Asthma & $28,6 \%(4 / 14)$ & $85.7 \%(12 / 14)$ & $0.006^{*}$ \\
\hline AERD & $14.3 \%(2 / 14)$ & $64.3 \%(9 / 14)$ & $0.018^{*}$ \\
\hline Eosinophils (count) & $300(200)$ & $300(500)$ & 0.603 \\
\hline Eosinophils (\%) & $4.90(4.1)$ & $4.90(3.8)$ & 0.982 \\
\hline $\lg A$ & $265.50(155)$ & $280.00(114)$ & 0.946 \\
\hline Normal lgE & $78.6 \%(11 / 14)$ & $28.6 \%(4 / 14)$ & $0.021^{*}$ \\
\hline $\lg \mathrm{E}$ & $111.00(69)$ & 282.00 (339) & $0.035^{*}$ \\
\hline $\lg G$ & $1327.50(473)$ & $1101.00(347)$ & 0.194 \\
\hline $\lg M$ & $117(35)$ & $120(122)$ & 0.701 \\
\hline
\end{tabular}

NERD - Aspirin Exacerbated Respiratory Disease; Lund-Kennedy - score for nasal endoscopic evaluation; Lund-Mackay - score for CT scan CRS evaluation; md (IQR) - median (interquartile range); NOSE - Nasal Obstruction Symptom Evaluation; NS - Nasal steroid; SNOT-20 - Sino-Nasal Outcome Test20

IgE levels ${ }^{(23)}$.

The EPOS2012 position paper noted that doxycycline has the potential to be a promising treatment alternative and that further studies were warranted ${ }^{(1)}$. Van Zele et al. ${ }^{(20)}$ were the first to publish a placebo-controlled study that showed a consistent effect of doxycycline, methylprednisolone, and placebo in a 3-week course after 20 days of surgery. Doxycycline was prescribed for a shorter period than in our study, which might explain the smaller improvement in endoscopic scores and fact that only postnasal drip was significantly reduced, with no effect on other symptoms. On the other hand, we did not analyze whether a sustained effect is observed after doxycycline treatment; further studies are needed to address this.

Nasal topical steroids are an excellent treatment option supported by high-quality evidence for patients with CRSwNP, but some patients do not experience satisfactory control of disease after this treatment ${ }^{(1)}$. According to EPOS, patients are conside- red to have difficult-to-treat CRSWNP when they do not show adequate disease control after surgery with use of nasal steroids and two short courses of antibiotics or systemic corticosteroids in the last year ${ }^{(1)}$. These patients might require alternative treatment, and doxycycline would be an excellent option in this setting, providing a satisfactory response in about half of patients (or more, if patients are well selected).

The most important pitfall of this study was the absence of double-blinded groups, but the major outcome measures were QoL questionnaires and endoscopic scores, which are less subject to bias. Most patients in this study were waitlisted for new sinus surgery; thus, if any patients were motivated to answer the QoL questionnaire improperly, their bias would probably be to show less improvement. None of our patients experienced any major adverse event, and none withdrew from the study. This achievement was probably due to efficient asthma control in all patients before the study and to maintenance of appropriate 
treatment during the study.

In the future, new clinical trials of long-term doxycycline therapy, with follow-up after treatment conclusion, assessment of clinical QoL outcomes, and measurement of markers of inflammation in nasal secretions and sinus mucosa, could elucidate the role of this drug in this patient population.

\section{Conclusion}

In conclusion, long-term low-dose doxycycline therapy may be indicated in patients with CRSWNP who do not experience clinical improvement despite conservative and surgical treatment, especially in patients without asthma or NERD and with normal $\operatorname{lgE}$ and eosinophil levels.

\section{Authorship contribution}

Acquisition, analysis, and interpretation of data for the work: ACPBS, TFPB; Drafting the work and revising it critically for important intellectual content: ACPBS, TFPB, RP, TRTA, RMP, FRP, PG, TZ, CB, RLV; Conception and design of the work: TFPB, RP, PG, $\mathrm{CB}, \mathrm{RLV}$; Interpretation of data for the work: RP, TZ; Analysis and interpretation of data for the work: PG, TZ, CB, RLV; All authors gave final approval of the version to be published

\section{Conflict of interest}

No conflict of interest.

\section{References}

1. Fokkens WJ, Lund VJ, Mullol J, et al. European Position Paper on Rhinosinusitis and Nasal Polyps 2012. Rhinol Supp. 2012 (23): 3, 1-298.

2. Pleis JR, Ward BW, Lucas JW. Summary health statistics for U.S. adults: National Health Interview Survey, 2009. National Center for Health Statistics. Vital Health Stat 10 2010; (249): 1-207.

3. Hastan D, Fokkens WJ, Bachert C, et al. Chronic rhinosinusitis in Europe - an underestimated disease. A GA2LEN study. Allergy. 2011;66(9):1216-23.

4. Pilan RR, Pinna FR, Bezerra TF, et al. Prevalence of chronic rhinosinusitis in Sao Paulo. Rhinology 2012; 50(2): 129-138.

5. Gliklich RE, Metson R. The health impact of chronic sinusitis in patients seeking otolaryngologic care. Otolaryngol Head Neck Surg 1995; 113: 104-109.

6. Szczeklik A, Nizankowska E, Sanak M, et al. Aspirin-induced rhinitis and asthma Current Opin Allergy Clin Immunol 2001; 1: $27 \mathrm{n} / \mathrm{m}$

7. Pezato R, ŚR, to 1: 27n Immu M, Ni, Ni 1: $27 n$ Immunolu $E$, et al. Role of imbalance of eicosanoid pathways and staphylococcal superantigens in chronic rhinosinusitis. Allergy 2012; 67(11):1347-1356.

8. Van Zele T, Claeys S, Gevaert P, et al. Differentiation of chronic sinus diseases by measurement of inflammatory mediators Allergy 2006; 61(11): 1280; feren

9. Bachert C, Gevaert P, Van Cauwenberge P, et al. Staphylococcus aureus superantigens and airway disease. Curr Allergy Asthma Rep. 2002;2(3):252-8.

10. Van Bruaene N, Bachert C. Tissue remodeling in chronic rhinosinusitis. Curr Opin Allergy Clin Immunol 2011; 11(1): 8-11.

11. Watelet JB, Bachert C, Claeys C, Van Cauwenberge P. Matrix metalloproteinases MMP-7, MMP-9 and their tissue inhibitor TIMP-1: expression in chronic sinusitis vs nasal polyposis. Allergy. 2004;59(1):54-60.

12. Foreman A, Holtappels G, Psaltis AJ, et al. Adaptive immune responses in Staphylococcus aureus biofilmbiofilmfilmus biofilmAllergy. 2011; 66(11):1449-56..

13. Van Zele T, Gevaert P, Watelet JB, et al. Staphylococcus aureus colonization and $\lg \mathrm{E}$ antibody formation to enterotoxins is increased in nasal polyposis. J Allergy Clin Immunol. 2004;114(4):981-3.

14. Akdis CA, Bachert C, Cingi C, et al. Endotypes and phenotypes of chronic rhinosinusitis: a PRACTALL document of the European Academy of Allergy and Clinical Immunology and the American Academy of Allergy, Asthma, Immunology. J Allergy Clin Immunol 2013; 131(6): 1479-1490.

15. Tan NC-W, Foreman A, Jardeleza C, et al. Intracellular Staphylococcus aureus: the Trojan horse of recalcitrant chronic rhinosinusitis? Int Forum Allergy Rhinol 2013; 3: 261.

16. Bezerra TF, Padua FG, Gebrim EM, et al. Biofilms in chronic rhinosinusitis with nasal polyps. Otolaryngol Head Neck Surg 2011; 144(4): 612-616

17. Foreman A, Wormald PJ. Different Biofilms, Different Disease? A Clinical Outcomes Study. Laryngoscope. 2010;120(8):1701-6.

18. Bachert C, Zhang N, Holtappels G, et al. Presence of IL-5 protein and IgE antibodies to staphylococcal enterotoxins in nasal polyps is associated with comorbid asthma. J Allergy Clin Immunol 2010; 126: 962-968.

19. Rosenblatt JE, Barrett JE, Brodie JL, et al. Comparison of in vitro activity and clinical pharmacology of doxycycline with other tetracyclines. Antimicrob agents chemother 1966; 6: 134-41

20. Van Zele T; Gevaert P, Holtappels G, et al. Oral steroids and doxycycline: Two different approaches to treat nasal polyps. J Allergy Clin Immunol 2010; 125: 1069-1076.

21. Yancey RJ, Sanchez MS, Ford CW. Activity of antibiotics against Staphylococcus aureus within polymorphonuclear neutrophils. Eur J Clin Microbiol Infect Dis 1991; 10(2): 107113.

22. Pezato R, Voegels RL, Pinto Bezerra TF, et al. Mechanical disfunction in the mucosal oedema formation of patients with nasal polyps. Rhinology 2014; 52(2): 162-166.

23. Wallwork B, Coman W, Mackay-Sim A, et al. A double-blind, randomized, placebo-controlled trial of macrolide in the treatment of chronic rhinosinusitis. Laryngoscope 2006; 116(2): 189-193.

24. Piccirillo JF, Merritt MG Jr, Richards ML. Psychometric and clinimetric validity of the 20-Item Sino-Nasal Outcome Test (SNOT20). Otolaryngol Head Neck Surg 2002; 126(1): 41-47.

25. Bezerra, TFP; Piccirillo, JF; Fornazieri, et al. Cross-Cultural Adaptation and Validation of SNOT-20 in Portuguese. Int J Otolaryngol. 2011;2011:306529.

26. Stewart MG, Witsell DL, Smith $T L$, et al. Development and validation of the Nasal Obstruction Symptom Evaluation (NOSE) scale. Otolaryngol Head Neck Surg 2004; 130: 157-163.

27. Bezerra, TFP; Padua, FGM;Pilan, RRM; et al. Cross-cultural adaptation and validation of a Quality of Life questionnaire: The Nasal Obstruction Symptom Evaluation questionnaire. Rhinology 2011; 49(2): 227-231.

28. Lund VJ; Kennedy DW. Staging for rhinosinusitis. Otolaryngol Head Neck Surg 1997; 117: S35-S40

29. Lund VJ; Mackay IS. Staging in rhinosinusitus. Rhinology 1993; 31(4): 183-184.

Thiago Freire Pinto Bezerra

Hospital das Clínicas (UFPE)

Av. Prof. Moraes Rego

S/N - Bloco "A" (Térreo)

Cidade Universitária

Recife - PE, 50.670-420

Brazil

E-mail: bezerratfp@gmail.com 